\title{
Modelación desde la perspectiva de la educación matemática crítica. Cuestiones relacionadas con la obsolescencia ${ }^{1}$
}

\author{
Modeling from the perspective of critical mathematics \\ education. Issues related to obsolescence
}

Modelando a partir da perspectiva da educação matemática crítica. Questões relacionadas com a obsolescência

Recibido: mayo 2013

Aceptado: agosto 2013
Diana Mirella Martínez Pinilla

Olga Patricia Páez $z^{3}$

Gloria García ${ }^{4}$

\section{Resumen}

En esta ponencia, describimos los procedimientos que hemos seguido en el montaje de un escenario de modelación desde la perspectiva socio-critica (Barbosa, 2006), en una clase de matemáticas de grado octavo de la educación básica. En particular ponemos el acento en las dificultades que presenta la modelación, desde esta perspectiva para integrarse al currículo de matemáticas del grado octavo y como parte de las prácticas en la clase de matemáticas. Especialmente el uso de modelos matemáticos en la sociedad, como es el caso del estudio de la obsolescencia y sus actuaciones para estudiarla en el currículo de grado octavo. Este fenómeno esta relacionando con las nuevas cultura juveniles por lo que esperamos lograr reflexiones relevantes y critica de los estudiantes.

Palabras clave: RJuego de rol; realidad; aprendizaje; procesos cognitivos; modelización; enseñanza; metodología de trabajo en el aula; sistema financiero; interés compuesto.

\begin{abstract}
In this paper, we describe the procedures we have followed in mounting a modeling scenario from the perspective of socio-critical (Barbosa, 2006), in a math class eighth grade of basic education. In particular we focus on the difficulties of modeling, from this perspective to integrate the curriculum eighth grade math and as part of the practices in the mathematics classroom. Especially the use of mathematical models in society, such as the study of obsolescence and their performances for study in the eighth grade curriculum. This phenomenon is interacting with the new youth culture so we hope to achieve relevant and critical thinking of students.
\end{abstract}

1 Artículo de Investigación.

2 Universidad Pedagógica Nacional, Maestría en Docencia de la Matemática. Contacto: mdma_dmartinez240@pedagogica.edu.co

3 Universidad Pedagógica Nacional, Maestría en Docencia de la Matemática. Contacto: mdma_opaez@pedagogica.edu.co

4 Universidad Pedagógica Nacional, Maestría en Docencia de la Matemática. Contacto: aprendizajeyevaluacion@gmail.com 
Keywords: learning, cognitive processes, mathematical modeling, learning environments, criticism, research and innovation in mathematics education, theoretical frameworks, learning theories: sociocultural located.

\section{Resumo}

Neste trabalho, descrevemos os procedimentos que seguimos na montagem de um cenário de modelagem a partir da perspectiva sócio-crítica (Barbosa, 2006), em uma aula de matemática da oitava série do ensino fundamental. Em particular, vamos nos concentrar nas dificuldades de modelagem, a partir dessa perspectiva de integrar o currículo de matemática da oitava série e como parte das práticas em sala de aula de matemática. Especialmente o uso de modelos matemáticos na sociedade, tais como o estudo da obsolescência e suas performances para o estudo no currículo oitava série. Este fenômeno está interagindo com a nova cultura da juventude de modo que esperamos alcançar pensamento relevante e crítica dos estudantes.

Palavras-chave: aprendizagem, processos cognitivos, modelagem matemática, os ambientes de aprendizagem, crítica, investigação e inovação em educação matemática, referenciais teóricos, as teorias de aprendizagem: sociocultural localizado.

\section{Introduccion}

Nuestro trabajo tiene como propósito, presentar la experiencia realizada durante el desarrollo del trabajo de grado de la Maestría en docencia de la Matemática, de la Universidad Pedagógica Nacional, donde se analizo el papel de la educación matemática en la formación de jóvenes críticos, específicamente en su relación con el consumo, mediante el desarrollo de actividades que involucran la modelación. La aproximación a las prácticas de consumo en los estudiantes, las limitamos en al consumo de los teléfonos celulares, puesto que hacen parte de la actual cultura juvenil. En esta aproximación hay una cierta familiaridad de los estudiantes (Monteiro, 1991), con las situaciones de compra de celulares y cambios de modelos.

Con base en esta familiaridad, revisamos el currículo escolar, la propuesta de contenidos de grado octavo y las experiencias de los estudiantes en la clase de matemáticas con actividades de modelación conectadas a situaciones reales, relacionándolas con el tema de variación. Respecto al currículo de la institución donde se realizo la actividad, encontramos que esta organizado por un listado de temas y contenidos de manera tradicional. Coherente con esta propuesta los ambientes de la clase de matemáticas, se inscriben en el paradigma del ejercicio (Skovsmose, 2000) Por tal razón el aprendizaje de ser crítico se constituye en una de las cuestiones fundamentales de este proyecto.

El primer encuentro temático de los estudiantes con el consumo de celulares lo propusimos desde la reflexión del fenómeno de la obsolescencia.

\section{Marco de referencia conceptual}

Acerca de la educación matemática crítica. La educación matemática crítica (EMC), se preocupa por el desarrollo de micro sociedades a través de aspectos democráticos, para Skovsmose (2000), estos aspectos están presentes en el aprendizaje de las matemáticas cuando se reflexiona sobre cada uno de los asuntos que hacen parte el salón de clase. Esto hace que las matemáticas tomen un lenguaje poderoso, que permite producir nuevos proyectos dentro del entorno que se está. Por lo cual, el conocer reflexivo 
de las matemáticas, que plantea Skovsmose (1999) permite identificar las nociones y comprensiones a través del modelaje matemático, haciendo uso del los lenguajes y el dialogo. Lo anterior se relaciona con la alfabetización bajo la perspectiva de la EMC, puestos que se debe potenciar a los estudiantes para revelar, la naturaleza crítica de la sociedad en la que viven y transforman, contribuyendo a una sociedad más democrática, (Skovsmose, O, 1999).

La formación y el conocimiento matemático es una herramienta útil para que los estudiantes puedan interactuar como miembros de la sociedad (Barbosa, 2006) y puedan aportar en la solución de situaciones sociales críticas, donde encuentren sentido a las matemáticas y reconozcan su poder formativo. De la misma forma, los Estándares Básicos de Competencias en Matemáticas (MEN, 2006), afirman que la educación matemática debe responder a los desafíos nacionales y mundiales, a la atención a la diversidad, a la interculturalidad y a la formación de ciudadanos y ciudadanas con las competencias necesarias para el ejercicio de sus derechos y deberes democráticos.

La perspectiva crítica nos permitió, construir una manera diferente de conectar situaciones del entorno de nuestros estudiantes, incorporando aspectos democráticos, que fundamenta en la posibilidad de crear un lenguaje con nuevas visiones, sobre las matemáticas escolares y el desarrollo de una ciudadanía critica. Finalmente, la educación matemática crítica debe permitir que los ciudadanos ejerzan las competencias democráticas, y el poder formativo de las matemáticas para tomar posiciones críticas, a través del conocer reflexivo mediante el modelaje matemático. (Skovsmose, O, 1999)

Figura 1: Esquema del proceso (Biembengut \& Hein, 1997).

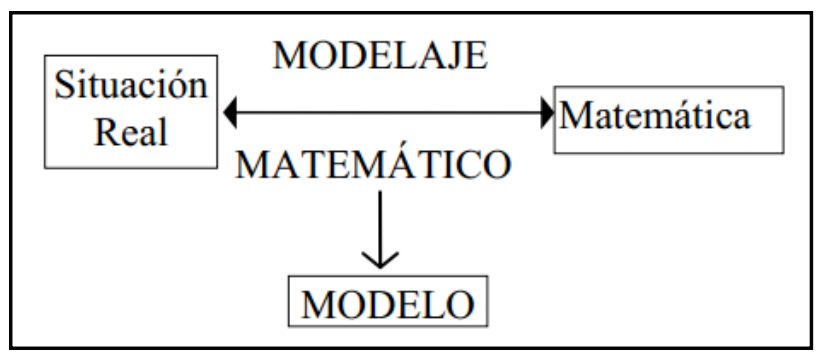

Fuente: Elaboración propia
Acerca de la modelación. La modelación matemática ha sido difundida especialmente a raíz de las críticas de la matemática moderna. La modelación en el contexto de la educación matemática, puede ser comprendida como un camino para el proceso de la enseñanza y el aprendizaje de las matemáticas partiendo de la realidad o contexto de los estudiantes.

Barbosa (2001)plantea como definición de modelación matemática desde la perspectiva socio - critica que es "un ambiente de aprendizaje que nos convida a los estudiantes a consultar y/o investigar, por medio de la matemática, con situaciones de referencia en la realidad (pág. 856)" esta definición expresa que la modelación matemática es "el arte de transformar problemas de la realidad en problemas matemáticos y resolverlos desde el lenguaje del mundo real". Autores como Burak (1992), afirman que la modelación es un cambio de modelo de la educación tradicional de enseñanza, a otra que busca que los problemas sean elegidos, en primer lugar y luego los contenidos matemáticos se usen para resolverlos.

En 1998, el Ministerio de Educación Nacional (MEN) de Colombia definió que uno de los procesos que se deben desarrollar en el área de matemáticas, es la modelación, para poder representar esquemas que se repiten en situaciones cotidianas, científicas y matemáticas y se puedan reconstruirlas mentalmente. En ese sentido la modelación es el punto de partida de una situación problemática real, de manera que las situaciones cotidianas y problemáticas, son un acercamiento a las matemáticas en contexto, en donde se pueda poner en práctica el aprendizaje activo, el desarrollo de pensamiento y que contribuyan a dar sentido a las matemáticas, para que los estudiantes modelen situaciones cotidianas.

Para Costa, Caldeira, \& Santos, (2011), un trabajo con modelación en el aula de clase, con base a situaciones de interés de los estudiantes, están posibilitándolos para comprender el papel de la matemática en la sociedad, que hace parte de su día a día, donde la modelación forma parte de su aprendizaje en la vida, para leer el mundo y comprender el poder de la toma de decisiones. Así mismo, cuando se trabaja en modelación en Educación Matemática Critica los profesores y estudiantes son participantes de 
su proceso de aprendizaje, no solo en el área de las matemáticas, sino también en cuestiones relacionadas con lo cotidiano, que sean de relevancia social y donde los aspectos estén relacionados con los intereses de los estudiantes.

Para iniciar el proceso de modelación en Educación Matemática Crítica, se debe generar problemas de investigación que no tengan una única respuesta o que haya muchos caminos hasta alcanzar. La modelación matemática es un camino para que los estudiantes aprendan a ser ciudadanos críticos y comprometidos en la construcción del conocimiento. Según Barbosa, (2001) la metáfora de la modelación como crítica, es vista como medio para criticar los modelos matemáticos en la sociedad, desde el aprendizaje de conceptos matemáticos y el desarrollo de "competencias de modelación". Para nuestra investigación los estudiantes conectaran las ideas de modelación con el impacto ambiental que genera el consumo de celulares en nuestro país. Este énfasis se relaciona con la idea de que la educación matemática debe participar en los esfuerzos para educar a los estudiantes a ser críticos y ciudadanos comprometidos.

\section{Metodología y análisis de datos}

La metodología de nuestra investigación es de tipo cualitativa, porque se busca respuestas a preguntas que hacen hincapié en situaciones críticas, que están presentes en la vida de nuestros estudiantes, estas experiencias sociales se establece y dan significado a ellos, así como las observaciones establecen diálogos en los estudiantes con una postura crítica, cuando desarrollan la tarea propuesta.

En primer lugar, se identifican las prácticas escolares relacionadas con las matemáticas, de los estudiantes de grado octavo y la profesora. En segundo lugar, se realizan actividades de interpretación de gráfi$\cos$, que contribuyan a la comprensión y a encontrar caminos de solución en tareas relacionadas con la variación. En tercer lugar, registramos en los diarios de campo, videos y narrativas los acontecimientos que se dan durante la ejecución y producción de los estudiantes cuando se les propone la tarea de modelación, para generar una posición crítica sobre la importancia del consumo acertado del teléfono celular, situación que fue complementada con un lectura de un folleto sobre la vida útil y el reciclaje adecuado de un teléfono celular. Y por ultimo con base en los registros obtenidos, se tomarán decisiones en torno al aprendizaje de los estudiantes, para luego ser publicadas para beneficio de la comunidad educativa.

\section{Conclusiones}

El desarrollo de estas actividades en grado octavo, ha generado en los estudiantes una posición crítica, respecto a la contaminación de residuos tecnológicos y su impacto en el medio ambiente. El desarrollo de las actividades de modelación en el aula, ocasiono algunas dificultades en los profesores, por su poco conocimiento de los aspectos de modelación matemática. Así mismo la inclusión de temas que no correspondían a lo planeado dentro del currículo de grado octavo, genero ciertas dificultades para realizar las actividades planteadas.

\section{Referencias}

Barbosa. (2006). Mathematical Modelling in classroom: a sócio-critical and discursive perspective. Zentralblatt für Didaktik der Mathematik, 38 (3), 293 - 301.

Barbosa, J. (2001). Modelagem na Educação Matemática: contribuições para o debate teórico. reunião anual da anped.

Biembengut, M., \& Hein, N. (1997). Modelo, modelación y modelaje. Epsilon: revista de la sociedad Andaluza de Educación Matemática “Thales" (38), 209-222.

Burak, D. (1992). Modelagem Matemática: uma metodologia alternativa para o ensino da Matemática. Campinas: Universidade Estadual de Campinas. 
Costa, J., Caldeira, A., \& Santos, A. (2011). A modelagem na Educação Matemática. En J. Costa, A. Caldeira, \& A. Santos, Modelagem em Educação Matemática (págs. 107-110). Belo Horizonte: Autentica.

Luna, A., \& Alves, J. (2007). Modelagem matemática: as interações discursivas de crianças da $4^{a}$ série a partir de um estudo sobre anorexia. In Anais da V Conferência Nacional sobre Modelagem na Educação Matemática (págs. 855-876). Ouro Preto, Minas Gerais, Brasil: Universidade Federal de Ouro Preto/ Universidade Federal de Minas Gerais.

MEN. (2006). Estándares Básicos de Competencias en Matemáticas. En Estándares Básicos de
Competencias en Lenguaje, Matemáticas, Ciencias y Ciudadanas (págs. 46 - 95). Bogotá: Ministerio de Educación Nacional.

Monteiro, A. (1991). Ensino de Matemática para Adultos através do Método Modelagem Matemática. Rio Claro: Instituto de Geociências e Ciências .

Skovsmose, O. (1999). Hacia una Filosofia de la Educación Matemática Crítica Bogotá. Bogotá: Empresa Docente.

Skovsmose, O. (2000). Escenarios de investigación. Revista EMA , 6 (1), 3-26. 